\title{
Sciendo
}

\section{Interdisciplinary groups perception on impact of technology: A study in Petro-Chemical sector}

\author{
Amit, KUNDU, \\ School of Management, Techno India Group, DN-25, \\ Sector V Salt Lake Kolkata- 700 091West Bengal; India \\ amitkundu@technoindiagroup.in, amit.kundu74@gmail.com
}

Ramanuj, MAJUMDAR,

Faculty of Marketing, Indian Institute of Management Calcutta, Diamond Harbour Road P.O. Joka, Kolkata - 700104 West Bengal; India ramanuj@iimcal.ac.in

\begin{abstract}
In today's competitive business environment firms who can afford to invest on superior technology are in general found to be more efficient. In technology driven industries companies tend to encash upon the benefits of the superior technology, engage in continuous innovation and invest in R\&D to gain competitive advantage. Notwithstanding this phenomenon, adoption of superior technology by any industry is extremely challenging. Even, Small and Medium size Enterprises (SMEs) in a developing country are often incapable of adopting the latest technology. Because they are expensive and moreover it leads to disruption to the traditional way of doing business and even different managerial functions differ on the adoption of technology.

This research paper collected perception regarding impact of technology by three broad managerial functions such as Operations, Marketing, Product development \& Innovation of a large Petrochemical company. With the help of Conjoint Analysis, Part worth and Ideal Achievement Index (Al) have been estimated. These estimates enable us to assess the perception of three inter disciplinary managerial groups regarding impact of technology on the downstream units (nearly 500 companies) of a Petro-Chemical Company in India.
\end{abstract}

Key Words: Technology, Achievement Index, Small and Medium size Enterprises (SME), Petrochemical Industry

JEL Classification: L26; 032 


\section{Introduction}

Haldia Petrochemical Limited (HPL) a joint venture undertaking of the Government of West Bengal, The Chatterjee Group and the Tata Group, located at $125 \mathrm{Km}$ from Kolkata, is a state of-the-art integrated Petrochemical Complex which commenced commercial production in August 2001.

HPL has actually catalyzed economic growth of the region by channeling significant funding in downstream processing industries. Through strategic market focus, innovative product application development and superlative customer support services, HPL has set up more than 500 downstream processing industries in West Bengal. With dedicated efforts, HPL truly symbolizes its motto - "Come Grow with Us".

In Petrochemical industry, companies tend to bank upon the benefits of the superior technology, engage in continuous innovation and invest in R\&D to gain competitive advantage. Notwithstanding this phenomenon, adoption of superior technology is extremely challenging. Further, Small and Medium size Enterprises (SMEs) in a developing country like India are often found to be incapable to adopt latest technology.

The present research study examines the perception of different managerial functions such as Operations, Marketing, R \& D of HPL regarding the impact of technology by the downstream units for their superior performance.

\section{Literature Review}

Globalization and the emergence of small and medium-sized enterprises (SMEs) are key worldwide trends (Gary Knight 2000). SMEs now provide a large share of current employment and future growth prospects in many countries worldwide (Organization for Economic Co-operation and Development, 1997). The success of SMEs under globalization depends in large part on the formulation and implementation of strategy (Porter, 1980). Globalization has made substantial changes in the market and the market becomes highly complex and uncertain. Entrepreneurial orientation, or entrepreneurship, reflects the firm's tendency to go for innovative, proactive, risky, independent, and competitively aggressive actions to achieve its strategic objectives (Dess, Lumpkin \& Covin 1997; Miller \& Friesen, 1984).

India's current economy after liberalization presents a unique mix of opportunities and challenges. Along with providing a proper environment for 
entrepreneurs to set up new ventures, it also provides a vast market for continued economic development. MSMEs contribute 8 percent of our GDP and promoting social and economic development through employment generation for more than 90 million people in more than 40 million enterprises (Ministry of MSME, Government of India, 2013). Over the past 20 years, it has grown at an average rate of about $6 \%$ a year. The rate has been consistently above $8 \%$ since 2003 and close to $9 \%$ in 2007 (Som, 2008).

Although most MSMEs are unregistered, the contribution of the sector to India's GDP has been growing consistently at 11 percent a year, which is higher than the overall GDP growth of 8 percent. Though the contribution of MSME sector is quite significant, there are a lot of barriers which have hindered its growth as per its full potential. (International Finance Corporation, World Bank Group, 2012).

MSMEs' contribution towards economic development is highly remarkable compared to any other sector of the economy. Its role towards investment, employment generation, exports, GDP, industrial production, etc. in every area is highly significant. All together the MSME segment accounts for more than 40 percent of the country's industrial output and more than 30 percent of exports. The overall contribution of this segment to India's Gross Domestic Product (GDP) has been around 8 percent per annum. However, many of the traditional problems areas of SMEs - financing, technology, managerial capabilities, productivity, and regulatory burdens - become more acute in a globalised, technology-driven environment. Fostering public-private partnerships and small-firm networks and clusters may be the most expeditious path to a dynamic SME sector (Organization for Economic Co-operation and Development, 2000).

Large firms in alliances with entrepreneurial firms gain access to new technologies, state-of- the-art engineering talent, and an overall inventive capability. Alliances with a large firm may give an entrepreneurial firm a social legitimacy not otherwise available to it (Alvarez \& Barney 2001).

\section{Research Objectives}

An attempt has been made to examine the issues associated with technology up-gradation by the downstream units of a Petro chemical Company (HPL) and measuring the organizational capabilities with regard to competitiveness as perceived by the senior managers of HPL.

The broad objectives of the present research can be stated as follows: 
1. To explore the relative importance of three broad functional areas, namely Marketing, Product development \& Innovation and Operations, with respect to absorption of new technology.

2. To estimate the relative degree of importance attached to each attribute within the three selected functional capabilities.

3. To estimate an Achievement Index (Al), measured by existing performance and ideal performance perceived by resource persons of HPL. This measures the performance gap and it can be effectively utilized to formulate a set of new policy framework.

\section{Research Methodology}

\subsection{Sources of Data}

The three experimental units namely Operations, Marketing, Product development \& Innovation are chosen. Some of the key senior managerial personnel of these three functional areas were surveyed using a structured questionnaire and their opinions regarding the chosen three research objectives were collected.

\subsection{Measurement Tools}

The attributes that were identified and the different levels of capability parameters have been taken into consideration for measuring the extent of adaptation of technology to be competitive in the volatile technological environment. The individual perception of the possible combination of attributes for measuring the readiness of accepting the technological change is considered as rating point (Rank).

The different levels of identified attributes of capability parameters are considered for measuring adaptation of technology know-how in the downstream units have been indicated in the table $\mathrm{I}$.

Table 1: Different levels of Capability parameters

\begin{tabular}{llccc}
\hline \multicolumn{1}{c}{ Attribute } & \multicolumn{3}{c}{ Levels } \\
\hline [A1] & Technology Branding & High & Medium & Low \\
& & T1 & T2 & T3 \\
[A2] & Marketing Linkages & High & & Low \\
& & M1 & & M2 \\
& & & & Low
\end{tabular}


[B2] Flexibility in Production System

[C1] Integrated product design for marketing

[C4] Internal environment
F1

High

P1

High

D1

Favourable

V1
F2

F3

Low

P2

$\begin{array}{cc}\text { Medium } & \text { Low } \\ \text { D2 } & \text { D3 } \\ & \text { Not } \\ & \text { favourable } \\ & \text { V2 }\end{array}$

The perception of each Managerial personnel of each experimental unit regarding the possible combination of attributes is recorded in the form of a preference vector and also the ranking for different combinations of the preference vector in a table suggested as below. The individual perception of the possible combination of the attributes for measuring the readiness of absorption of the technological change is considered as rating point (Rank). Out of 216 combinations following 10 combinations has been chosen (Ref Table 2).

Table 2. Possible Combination of attributes with their levels

\begin{tabular}{lll}
\hline & Serial No. & Combinations \\
\hline 1 & T3 M2 F3 P1D3 V1 \\
2 & T3 M1F2 P2D1 V1 \\
3 & T1 M1F2 P2D2 V2 \\
4 & T2 M2F1 P1 D1 V2 \\
5 & T1 M2F3 P2D1 V1 \\
6 & T3 M1F1 P2D3 V1 \\
7 & T1 M2F2 P2D2 V2 \\
8 & T2 M1F1 P2 D2 V2 \\
9 & T1 M2F3 P1D1 V2 \\
10 & T3 M1F2 P2D3 V1 \\
\hline
\end{tabular}

The estimation of the part-worth of the attributes such as Marketing, Product development \& Innovation and Operations and also the relative importance of the different levels of different attributes is done through Conjoint Analysis.

\section{Analysis \& Findings}

The researchers has prescribed the following mathematical model incorporating the different levels of the Capability Parameters, such as, Marketing, Product development \& Innovation and Operations. The Coefficients associated with them have been calculated by solving the following equation on the basis of observations on: 
(Rank, $T_{1}, T_{2}, M_{1}, F_{1}, F_{2}, P_{1}, D_{1}, D_{2}, V_{1}$ )

Rank $=\beta_{0}+\beta_{1} \mathrm{~T}_{1}+\beta_{2} \mathrm{~T}_{2}+\beta_{3} \mathrm{M}_{1}+\beta_{4} \mathrm{~F}_{1}+\beta_{5} \mathrm{~F}_{2}+\beta_{6} \mathrm{P}_{1}+\beta_{7} \mathrm{D}_{1}+\beta_{8} \mathrm{D}_{2}$ $+\beta_{9} \mathrm{~V}_{1}$ [1]

Where $T_{1}, T_{2}, M_{1}, F_{1}, F_{2}, P_{1}, D_{1}, D_{2}, V_{1}$ are the levels of attributes and $\beta_{1,}, \beta_{2} \ldots \beta_{9}$ are the corresponding coefficients.

The typical data set for $T_{1}, \ldots, \ldots, M_{1}, \ldots, F_{1}, \ldots, P_{1}, . . D_{1}, \ldots, V_{1}, \ldots$ would be as under.

For technological know-how of marketing capability,

Table 3. Dataset Format for Each Attribute

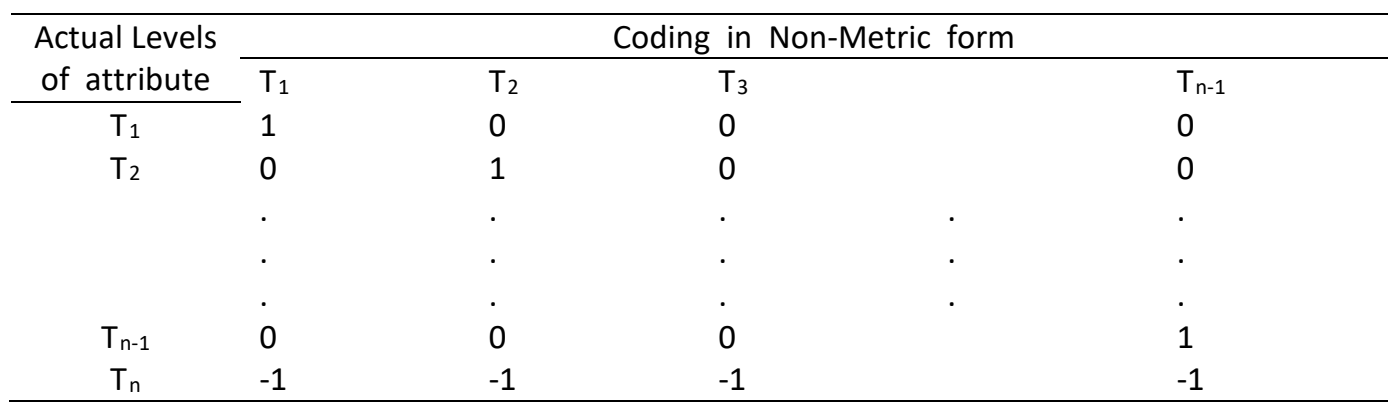

In this structure, the sum total of the Coefficients is assumed to be zero. Same assumption is applicable for other capability parameters.

After applying the above function to the data set, there would emerge 10 equations which are to be solved in order to find out the value of the coefficients shown in the function (Ref Table 3).

Based on the coefficients associated with the independent variables such as $T_{1}, T_{2}, T_{3}, M_{1}, M_{2}, F_{1}, F_{2}, F_{3}, P_{1}, P_{2}, D_{1}, D_{2}, D_{3}, V_{1}, V_{2}$, rescaled part-worth have been worked out for each experimental unit. Here, a scale is defined with the extreme values as 0 and 1 .

After having estimated part-worth of each level, total part worth for the combination of ranked one is calculated. It denotes the Existing estimated partworth of (E) as perceived by the concerned sub-unit considering present status of the downstream units. Each sub units of the concerned Experimental unit has also asked to provide the best possible combination of the levels of each attributes which would be Ideal (I) as perceived by the sub units for the sustainable growth of the downstream units in the present dynamic technological environment. The ratio of the two, exiting (E) and ideal (I) is defined as Achievement Index (Al). The 
significance of the Achievement Index (AI) is to measure the extent of operating performance of the SME units.

\section{Experimental Unit I - Marketing Division}

Based on the perceived data of the marketing unit in their three respective sub-divisions namely, export division, eastern region marketing division and business development rescaled part-worth have been estimated ( ref table 4 and table 5).

Table 4. Value of coefficients associated with the Levels of Capability Parameters

\begin{tabular}{|c|c|c|c|}
\hline \multirow{2}{*}{$\begin{array}{l}\text { Levels of } \\
\text { Design } \\
\text { Parameters }\end{array}$} & \multicolumn{3}{|c|}{ Value of coefficients associated with the Levels of Design Parameters } \\
\hline & $\begin{array}{c}\text { Export Division } \\
\text { [Experimental Unit] }\end{array}$ & $\begin{array}{c}\text { Eastern Region } \\
\text { Marketing Division } \\
\text { [Experimental Unit] }\end{array}$ & $\begin{array}{c}\text { Business } \\
\text { Development } \\
\text { [Experimental Unit] }\end{array}$ \\
\hline Constant & 5.333 & 5.333 & 6.000 \\
\hline $\mathrm{T}_{1}$ & 0.333 & 0 & -1.333 \\
\hline $\mathrm{T}_{2}$ & -2.667 & 0 & -0.333 \\
\hline$T_{3}$ & 2.334 & 0 & 1.667 \\
\hline $\mathrm{M}_{1}$ & 3.000 & 2.5 & 2.5 \\
\hline $\mathrm{M}_{2}$ & -3.000 & -2.5 & -2.5 \\
\hline $\mathrm{F}_{1}$ & 2.000 & 0 & 0.667 \\
\hline $\mathrm{F}_{2}$ & -2.000 & -1.000 & -0.333 \\
\hline $\mathrm{F}_{3}$ & 0 & 1.000 & -0.333 \\
\hline$P_{1}$ & 1.000 & 0 & 2.000 \\
\hline$P_{2}$ & -1.000 & 0 & -2.000 \\
\hline $\mathrm{D}_{1}$ & 2.333 & 2.667 & 2.667 \\
\hline$D_{2}$ & 2.333 & -3.333 & 0.667 \\
\hline$D_{3}$ & -4.667 & 0.667 & -3.334 \\
\hline$V_{1}$ & 0 & -3.5 & -0.5 \\
\hline$V_{2}$ & 0 & 3.5 & 0.5 \\
\hline
\end{tabular}

Table 5 Estimation of Rescaled Part-worth of the Capability Parameters

\begin{tabular}{|c|c|c|c|c|c|c|c|c|}
\hline & \multicolumn{2}{|c|}{$\begin{array}{l}\text { Part-worth[Export } \\
\text { Division] }\end{array}$} & & \multicolumn{2}{|c|}{$\begin{array}{lr}\text { Part-worth } & \text { [Eastern } \\
\text { Region } & \text { Marketing } \\
\text { Division] } & \end{array}$} & & \multicolumn{2}{|c|}{$\begin{array}{l}\text { Part-worth [Business } \\
\text { Development] }\end{array}$} \\
\hline & Original & Rescaled & & Original & Rescaled & & Original & Rescaled \\
\hline $\mathrm{T}_{1}$ & 0.333 & 0.652 & $\mathrm{~T}_{1}$ & 0 & 0.5 & $\mathrm{~T}_{1}$ & -1.333 & 0.333 \\
\hline $\mathrm{T}_{2}$ & -2.667 & 0.2608 & $\mathrm{~T}_{2}$ & 0 & 0.5 & $\mathrm{~T}_{2}$ & -0.333 & 0.5 \\
\hline$T_{3}$ & 2.334 & 0.913 & $\mathrm{~T}_{3}$ & 0 & 0.5 & $T_{3}$ & 1.667 & 0.8333 \\
\hline
\end{tabular}




\begin{tabular}{ccccccccc}
\hline $\mathrm{M}_{1}$ & 3.000 & 1 & $\mathrm{M}_{1}$ & 2.5 & 0.857 & $\mathrm{M}_{1}$ & 2.5 & 0.972 \\
$\mathrm{M}_{2}$ & -3.000 & 0.217 & $\mathrm{M}_{2}$ & -2.5 & 0.142 & $\mathrm{M}_{2}$ & -2.5 & 0.139 \\
$\mathrm{~F}_{1}$ & 2.000 & 0.869 & $\mathrm{~F}_{1}$ & 0 & 0.5 & $\mathrm{~F}_{1}$ & 0.667 & 0.667 \\
$\mathrm{~F}_{2}$ & -2.000 & 0.347 & $\mathrm{~F}_{2}$ & -1.000 & 0.357 & $\mathrm{~F}_{2}$ & -0.333 & 0.5 \\
$\mathrm{~F}_{3}$ & 0 & 0.6087 & $\mathrm{~F}_{3}$ & 1.000 & 0.643 & $\mathrm{~F}_{3}$ & -0.333 & 0.5 \\
$\mathrm{P}_{1}$ & 1.000 & 0.739 & $\mathrm{P}_{1}$ & 0 & 0.5 & $\mathrm{P}_{1}$ & 2.000 & 0.889 \\
$\mathrm{P}_{2}$ & -1.000 & 0.478 & $\mathrm{P}_{2}$ & 0 & 0.5 & $\mathrm{P}_{2}$ & -2.000 & 0.222 \\
$\mathrm{D}_{1}$ & 2.333 & 0.913 & $\mathrm{D}_{1}$ & 2.667 & 0.881 & $\mathrm{D}_{1}$ & 2.667 & 1 \\
$\mathrm{D}_{2}$ & 2.333 & 0.913 & $\mathrm{D}_{2}$ & -3.333 & 0.0238 & $\mathrm{D}_{2}$ & 0.667 & 0.667 \\
$\mathrm{D}_{3}$ & -4.667 & 0 & $\mathrm{D}_{3}$ & 0.667 & 0.595 & $\mathrm{D}_{3}$ & -3.334 & 0 \\
$\mathrm{~V}_{1}$ & 0 & 0.6087 & $\mathrm{~V}_{1}$ & -3.5 & 0 & $\mathrm{~V}_{1}$ & -0.5 & 0.472 \\
$\mathrm{~V}_{2}$ & 0 & 0.6087 & $\mathrm{~V}_{2}$ & 3.5 & 1 & $\mathrm{~V}_{2}$ & $0 / 5$ & 0.639 \\
\hline
\end{tabular}

Estimation of the Achievement Index for each sub-unit of the marketing divisions along-with weightage of the marketing strategies perceived by the individual sub-units has also been computed (ref Table 6).

Table 6. Estimation of Achievement Index (AI) as perceived by Marketing Division

\begin{tabular}{|c|c|c|c|c|}
\hline $\begin{array}{l}\text { Sub-units of } \\
\text { Marketing } \\
\text { Division }\end{array}$ & $\begin{array}{l}\text { Existing Part-Worth } \\
\text { with their sub value } \\
\text { ( E) }\end{array}$ & $\begin{array}{l}\text { Ideal Part-Worth } \\
\text { with their sub } \\
\text { value (I) }\end{array}$ & $\begin{array}{l}\text { Achievement } \\
\text { Index (AI) }\end{array}$ & $\begin{array}{l}\text { Importance Level } \\
\text { of Marketing } \\
\text { Strategies }\end{array}$ \\
\hline Export Division & $\begin{array}{c}\mathrm{T}_{3} \mathrm{M}_{2} \mathrm{~F}_{3} \mathrm{P}_{2} \mathrm{D}_{3} \mathrm{~V}_{1} \\
(3.0864)\end{array}$ & $\begin{array}{c}\mathrm{T}_{2} \mathrm{M}_{1} \mathrm{~F}_{2} \mathrm{P}_{1} \mathrm{D}_{3} \mathrm{~V}_{1} \\
(3.8685)\end{array}$ & 0.7978 & 45.83 \\
\hline $\begin{array}{l}\text { Eastern Region } \\
\text { Marketing } \\
\text { Division }\end{array}$ & $\begin{array}{c}\mathrm{T}_{1} \mathrm{M}_{2} \mathrm{~F}_{2} \mathrm{P}_{2} \mathrm{D}_{2} \mathrm{~V}_{2} \\
(2.5228)\end{array}$ & $\begin{array}{c}\mathrm{T}_{2} \mathrm{M}_{1} \mathrm{~F}_{1} \mathrm{P}_{1} \mathrm{D}_{1} \mathrm{~V}_{1} \\
(3.238)\end{array}$ & 0.779 & 25.0 \\
\hline $\begin{array}{l}\text { Business } \\
\text { Development }\end{array}$ & $\begin{array}{c}\mathrm{T}_{1} \mathrm{M}_{2} \mathrm{~F}_{2} \mathrm{P}_{2} \mathrm{D}_{2} \mathrm{~V}_{2} \\
(2.5)\end{array}$ & $\begin{array}{c}\mathrm{T}_{2} \mathrm{M}_{1} \mathrm{~F}_{2} \mathrm{P}_{1} \mathrm{D}_{2} \mathrm{~V}_{1} \\
(3.4)\end{array}$ & 0.735 & 40.0 \\
\hline
\end{tabular}

\section{Experimental Unit II- Product Development and Innovation}

Based on the perceived data of the Product Development and Innovation unit in their four respective sub-divisions namely, Market Research Group, New Product Innovation Team, Application-Research -Development( ARD) Team and Entrepreneurs Training and Development Team of Eastern region Market of Haldia Petrochemicals Limited, rescaled part-worth have been estimated (ref Table 7 and Table 8). 
Table 7. Value of coefficients associated with the Levels of Capability Parameters

\begin{tabular}{|c|c|c|c|c|}
\hline \multirow[b]{2}{*}{$\begin{array}{l}\text { Levels of } \\
\text { Design } \\
\text { Parameters }\end{array}$} & \multicolumn{4}{|c|}{ Value of coefficients associated with the Levels of Design Parameters } \\
\hline & $\begin{array}{l}\text { Market } \\
\text { Research } \\
\text { Group } \\
\text { [Experimental } \\
\text { Unit] }\end{array}$ & $\begin{array}{l}\text { New Product } \\
\text { Innovation } \\
\text { Team } \\
\text { [Experimental } \\
\text { Unit] }\end{array}$ & $\begin{array}{c}\text { Application-Research - } \\
\text { Development( ARD) } \\
\text { Team [Experimental } \\
\text { Unit] }\end{array}$ & $\begin{array}{l}\text { Entrepreneurs } \\
\text { Training and } \\
\text { Development } \\
\text { Team } \\
\text { [Experimental Unit] }\end{array}$ \\
\hline Constant & 5.167 & 4.333 & 6.500 & 5.167 \\
\hline $\mathrm{T}_{1}$ & -0.667 & -5.000 & 1.333 & -0.333 \\
\hline $\mathrm{T}_{2}$ & -0.667 & -1.000 & 1.333 & -2.333 \\
\hline$T_{3}$ & 1.334 & 6.000 & -2.667 & 2.667 \\
\hline$M_{1}$ & 1.5 & 0.500 & 3.000 & 1.5 \\
\hline $\mathrm{M}_{2}$ & -1.5 & -0.500 & -3.000 & -1.5 \\
\hline $\mathrm{F}_{1}$ & 2.667 & -0.333 & 1.667 & 2.000 \\
\hline $\mathrm{F}_{2}$ & -1.333 & -2.333 & -0.333 & -1.000 \\
\hline $\mathrm{F}_{3}$ & -1.333 & 2.667 & -1.333 & -1.000 \\
\hline $\mathrm{P}_{1}$ & -0.500 & -2.500 & 1.5 & 1.000 \\
\hline $\mathrm{P}_{2}$ & 0.500 & 2.500 & -1.5 & -1.000 \\
\hline $\mathrm{D}_{1}$ & 1.333 & 2.000 & -1.000 & 3.667 \\
\hline $\mathrm{D}_{2}$ & -0.667 & -1.000 & 0 & 0.667 \\
\hline $\mathrm{D}_{3}$ & -0.667 & -1.000 & 1.000 & -4.334 \\
\hline$V_{1}$ & 1.500 & -3.000 & 2.000 & -1.000 \\
\hline$V_{2}$ & -1.500 & 3.000 & -2.000 & 1.000 \\
\hline
\end{tabular}

Table 8. Estimation of Rescaled Part-worth of the Capability parameters

\begin{tabular}{|c|c|c|c|c|c|c|c|c|c|c|}
\hline \multicolumn{3}{|c|}{$\begin{array}{l}\text { Part-worth } \\
\text { [[Market } \\
\text { Research } \\
\text { Group] }\end{array}$} & \multicolumn{2}{|c|}{$\begin{array}{l}\text { Part-worth [New } \\
\text { Product } \\
\text { Innovation } \\
\text { Team] }\end{array}$} & & \multicolumn{2}{|c|}{$\begin{array}{l}\text { Part-worth } \\
\text { [Application- } \\
\text { Research - } \\
\text { Development( } \\
\text { ARD) Team] }\end{array}$} & & \multicolumn{2}{|c|}{$\begin{array}{l}\text { Experimental } \\
\text { Unit } \\
\text { [Entrepreneurs } \\
\text { Training and } \\
\text { Development } \\
\text { Team] }\end{array}$} \\
\hline Original & Rescaled & & Original & Rescaled & & Original & Rescaled & & Original & Rescaled \\
\hline-0.667 & 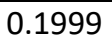 & $\mathrm{T}_{1}$ & -3 & U & $\mathrm{T}_{1}$ & 1.333 & 2 & 1 & $U$ & 0.500 \\
\hline-0.667 & 0.1999 & $\mathrm{~T}_{2}$ & -1.000 & 0.3636 & $\mathrm{~T}_{2}$ & 1.333 & 0.722 & $\mathrm{~T}$ & -2.333 & .25 \\
\hline 1.334 & 0.680 & $T_{3}$ & 6.000 & 1.000 & $T_{3}$ & -2.667 & 0.055 & $\mathrm{~T}$ & 2.667 & 0.875 \\
\hline 1.5 & 0.7199 & $M$ & 0.500 & 0.500 & $M$ & 3.000 & 1 & $\mathrm{M}$ & 1.5 & 0.73 \\
\hline-1.5 & 0 & $M$ & -0.500 & 0.409 & $M$ & -3.000 & 0 & $M$ & -1.5 & 0.354 \\
\hline 2.667 & 1 & $\mathrm{~F}_{1}$ & -0.333 & 0.42 & $\mathrm{~F}_{1}$ & 1.667 & 0.778 & $\Gamma$ & 2.00 & 0.792 \\
\hline-1.333 & 0.0400 & $F_{2}$ & -2.333 & 0.242 & $F_{2}$ & -0.333 & 0.4445 & $\mathrm{~F}$ & -1.000 & 0.4167 \\
\hline-1.333 & 0.0400 & $F_{3}$ & 2.667 & 0.697 & $F_{3}$ & -1.333 & 0.278 & 1 & -1.000 & 0.4167 \\
\hline-0.500 & 0.239 & $P_{1}$ & -2.500 & 0.227 & $P_{1}$ & 1.5 & 0.75 & $P$ & 1.000 & 0.667 \\
\hline 0.500 & 0.479 & $P_{2}$ & 2.500 & 0.6818 & $P_{2}$ & -1.5 & 0.25 & $P$ & -1.000 & 0.4167 \\
\hline
\end{tabular}




\begin{tabular}{cccccccccccc}
\hline $\mathrm{D}_{1}$ & 1.333 & 0.679 & $\mathrm{D}_{1}$ & 2.000 & 0.636 & $\mathrm{D}$ & -1.000 & 0.333 & $\mathrm{D}$ & 3.667 & 1 \\
$\mathrm{D}_{2}$ & -0.667 & 0.1999 & $\mathrm{D}_{2}$ & -1.000 & 0.3636 & $\mathrm{D}$ & 0 & 0.500 & $\mathrm{D}$ & 0.667 & 0.625 \\
$\mathrm{D}_{3}$ & -0.667 & 0.1999 & $\mathrm{D}_{3}$ & -1.000 & 0.3636 & $\mathrm{D}$ & 1.000 & 0.6667 & $\mathrm{D}$ & -4.334 & 0 \\
$\mathrm{~V}_{1}$ & 1.500 & 0 & $\mathrm{~V}_{1}$ & -3.000 & 0.1818 & $\mathrm{~V}_{1}$ & 2.000 & 0.8333 & $\mathrm{~V}$ & -1.000 & 0.4167 \\
$\mathrm{~V}_{2}$ & -1.500 & 0.7199 & $\mathrm{~V}_{2}$ & 3.000 & 0.727 & $\mathrm{~V}_{2}$ & -2.000 & 0.1667 & $\mathrm{~V}$ & 1.000 & 0.667 \\
\hline
\end{tabular}

Estimation of the Achievement Index for each sub-unit of the Product Development and Innovation along-with weightage of the innovation strategies perceived by the individual sub-units has also been computed (ref Table 9).

Table 9. Estimation of Achievement Index (AI) as perceived by Product Development and Innovation Unit

\begin{tabular}{|c|c|c|c|c|}
\hline $\begin{array}{l}\text { Sub-units of } \\
\text { Product } \\
\text { Development } \\
\text { and Innovation }\end{array}$ & $\begin{array}{l}\text { Existing Part- } \\
\text { Worth with } \\
\text { their sub value } \\
\text { ( } E)\end{array}$ & $\begin{array}{l}\text { Ideal Part-Worth with } \\
\text { their sub value (I) }\end{array}$ & $\begin{array}{l}\text { Achievement } \\
\text { Index (Al) }\end{array}$ & $\begin{array}{c}\text { Importance } \\
\text { Level of } \\
\text { Product } \\
\text { Development \& } \\
\text { Innovation } \\
\text { Strategies }\end{array}$ \\
\hline $\begin{array}{l}\text { Market } \\
\text { Research } \\
\text { Group }\end{array}$ & $\begin{array}{c}\mathrm{T}_{1} \mathrm{M}_{1} \mathrm{~F}_{2} \mathrm{P}_{2} \mathrm{D}_{2} \mathrm{~V}_{2} \\
(2.3586)\end{array}$ & $\mathrm{T}_{2} \mathrm{M}_{1} \mathrm{~F}_{1} \mathrm{P}_{2} \mathrm{D}_{2} \mathrm{~V}_{2}(3.3186)$ & 0.71 & 33.40 \\
\hline $\begin{array}{c}\text { New Product } \\
\text { Innovation } \\
\text { Team }\end{array}$ & $\begin{array}{c}\mathrm{T}_{1} \mathrm{M}_{2} \mathrm{~F}_{2} \mathrm{P}_{2} \mathrm{D}_{2} \mathrm{~V}_{2} \\
(2.4234)\end{array}$ & $\begin{array}{c}\mathrm{T}_{2} \mathrm{M}_{1} \mathrm{~F}_{1} \mathrm{P}_{2} \mathrm{D}_{1} \mathrm{~V}_{2} \\
(3.3354)\end{array}$ & 0.726 & 32.26 \\
\hline $\begin{array}{l}\text { Application- } \\
\text { Research - } \\
\text { Development( } \\
\text { ARD) Team }\end{array}$ & $\begin{array}{c}\mathrm{T}_{1} \mathrm{M}_{2} \mathrm{~F}_{2} \mathrm{P}_{2} \mathrm{D}_{2} \mathrm{~V}_{2} \\
(2.0832)\end{array}$ & $\begin{array}{c}\mathrm{T}_{2} \mathrm{M}_{1} \mathrm{~F}_{1} \mathrm{P}_{2} \mathrm{D}_{2} \mathrm{~V}_{1} \\
(4.0833)\end{array}$ & 0.51 & 22.7 \\
\hline $\begin{array}{l}\text { Entrepreneurs } \\
\text { Training and } \\
\text { Development } \\
\text { Team }\end{array}$ & $\begin{array}{c}T_{3} M_{2} F_{3} P_{1} D_{3} V_{1} \\
(2.7294)\end{array}$ & $\begin{array}{c}\mathrm{T}_{3} \mathrm{M}_{1} \mathrm{~F}_{1} \mathrm{P}_{1} \mathrm{D}_{1} \mathrm{~V}_{1} \\
(4.4807)\end{array}$ & 0.609 & 23.8 \\
\hline
\end{tabular}

\section{Experimental Unit III - Operations Division}

Based on the perceived data of the operations unit in their three respective sub-divisions namely, Materials Management, Production \& Operations and Logistics \& distribution rescaled part-worth have been estimated ( ref table 10 and table 11). 
Table 10. Value of coefficients associated with the Levels of Capability Parameters

Levels of

Design

Parameters

Materials

Management

[Experimental Unit]

\begin{tabular}{cccc} 
& [Experimental Unit] & [Experimental Unit] \\
\hline Constant & 6.833 & 3.333 & 8.000 \\
$\mathrm{~T}_{1}$ & 3.000 & -2.000 & 5.000 \\
$\mathrm{~T}_{2}$ & 2.000 & -3.000 & 1.000 \\
$\mathrm{~T}_{3}$ & -5.000 & 5.000 & -6.000 \\
$\mathrm{M}_{1}$ & 3.000 & 1.000 & 2.5 \\
$\mathrm{M}_{2}$ & -3.000 & -1.000 & -2.5 \\
$\mathrm{~F}_{1}$ & 2.333 & 1.333 & 2.667 \\
$\mathrm{~F}_{2}$ & 0.333 & -3.667 & -0.333 \\
$\mathrm{~F}_{3}$ & -2.667 & 2.334 & -2.333 \\
$\mathrm{P}_{1}$ & 2.5 & -4.000 & 5.5 \\
$\mathrm{P}_{2}$ & -2.5 & 4.000 & -5.5 \\
$\mathrm{D}_{1}$ & 0.333 & 3.333 & -0.667 \\
$\mathrm{D}_{2}$ & -1.667 & -5.667 & 2.333 \\
$\mathrm{D}_{3}$ & 1.334 & 2.334 & -1.667 \\
$\mathrm{~V}_{1}$ & 1.000 & -7.000 & 4.000 \\
$\mathrm{~V}_{2}$ & -1.000 & 7.000 & -4.000 \\
\hline
\end{tabular}

Table 11. Rescaled Part-worth corresponding to the different levels of the Capability parameters

\begin{tabular}{|c|c|c|c|c|c|c|c|}
\hline \multicolumn{2}{|c|}{$\begin{array}{c}\text { Part-worth[Materials } \\
\text { Management] }\end{array}$} & & \multicolumn{2}{|c|}{$\begin{array}{l}\text { Part-worth } \\
\text { [Production } \\
\text { Operations] }\end{array}$} & & \multicolumn{2}{|c|}{$\begin{array}{l}\text { Part-worth [Logistics \& } \\
\text { distribution }\end{array}$} \\
\hline Original & Rescaled & & Original & Rescaled & & Original & Rescaled \\
\hline 3.000 & 1 & $\mathrm{~T}_{1}$ & -2.000 & 0.357 & $\mathrm{~T}_{1}$ & 5.000 & 0.956 \\
\hline 2.000 & 0.875 & $\mathrm{~T}_{2}$ & -3.000 & 0.2857 & $\mathrm{~T}_{2}$ & 1.000 & 0.608 \\
\hline-5.000 & 0 & $\mathrm{~T}_{3}$ & 5.000 & 0.857 & $\mathrm{~T}_{3}$ & -6.000 & 0 \\
\hline 3.000 & 1 & $\mathrm{M}_{1}$ & 1.000 & 0.571 & $M_{1}$ & 2.5 & 0.739 \\
\hline-3.000 & 0.25 & $\mathrm{M}_{2}$ & -1.000 & 0.428 & $M_{2}$ & -2.5 & 0.304 \\
\hline 2.333 & 0.916 & $\mathrm{~F}_{1}$ & 1.333 & 0.595 & $\mathrm{~F}_{1}$ & 2.667 & 0.754 \\
\hline 0.333 & 0.667 & $\mathrm{~F}_{2}$ & -3.667 & 0.238 & $F_{2}$ & -0.333 & 0.492 \\
\hline-2.667 & 0.29 & $\mathrm{~F}_{3}$ & 2.334 & 0.667 & $F_{3}$ & -2.333 & 0.318 \\
\hline 2.5 & 0.9375 & $P_{1}$ & -4.000 & 0.214 & $P_{1}$ & 5.5 & 1 \\
\hline-2.5 & 0.3125 & $P_{2}$ & 4.000 & 0.785 & $\mathrm{P}_{2}$ & -5.5 & 0.043 \\
\hline 0.333 & 0.667 & $\mathrm{D}_{1}$ & 3.333 & 0.738 & $\mathrm{D}_{1}$ & -0.667 & 0.463 \\
\hline-1.667 & 0.4167 & $\mathrm{D}_{2}$ & -5.667 & 0.095 & $\mathrm{D}_{2}$ & 2.333 & 0.724 \\
\hline
\end{tabular}




\begin{tabular}{ccccccccc}
$\mathrm{D}_{3}$ & 1.334 & 0.791 & $\mathrm{D}_{3}$ & 2.334 & 0.667 & $\mathrm{D}_{3}$ & -1.667 & 0.376 \\
$\mathrm{~V}_{1}$ & 1.000 & 0.125 & $\mathrm{~V}_{1}$ & -7.000 & 0 & $\mathrm{~V}_{1}$ & 4.000 & 0.869 \\
$\mathrm{~V}_{2}$ & -1.000 & 0.5 & $\mathrm{~V}_{2}$ & 7.000 & 1 & $\mathrm{~V}_{2}$ & -4.000 & 0.174 \\
\hline
\end{tabular}

Estimation of the Achievement Index for each sub-unit of the operations divisions along-with weightage of the operational strategies perceived by the individual sub-units has also been computed (ref Table 12).

Table 12. Estimation of Achievement Index (Al) as perceived by Operations Division

\begin{tabular}{|c|c|c|c|c|c|}
\hline $\begin{array}{l}\text { Sub-units } \\
\text { Operations } \\
\text { Division }\end{array}$ & of & $\begin{array}{l}\text { Existing Part-Worth } \\
\text { with their sub value } \\
\text { ( E) }\end{array}$ & $\begin{array}{l}\text { Ideal Part-Worth } \\
\text { with their sub } \\
\text { value (I) }\end{array}$ & $\begin{array}{l}\text { Achievement } \\
\text { Index (Al) }\end{array}$ & $\begin{array}{l}\text { Importance } \\
\text { Level of } \\
\text { Operational } \\
\text { Strategies }\end{array}$ \\
\hline $\begin{array}{l}\text { Materials } \\
\text { Management } \\
\text { Division }\end{array}$ & & $\begin{array}{c}\mathrm{T}_{3} \mathrm{M}_{2} \mathrm{~F}_{3} \mathrm{P}_{1} \mathrm{D}_{3} \mathrm{~V}_{1} \\
(2.3935)\end{array}$ & $\begin{array}{c}\mathrm{T}_{2} \mathrm{M}_{1} \mathrm{~F}_{2} \mathrm{P}_{1} \mathrm{D}_{2} \mathrm{~V}_{1} \\
(4.02)\end{array}$ & 0.595 & 17.1 \\
\hline $\begin{array}{l}\text { Production } \\
\text { Operations }\end{array}$ & $\&$ & $\begin{array}{c}\mathrm{T}_{2} \mathrm{M}_{2} \mathrm{~F}_{1} \mathrm{P}_{1} \mathrm{D}_{1} \mathrm{~V}_{2} \\
(2.2607)\end{array}$ & $\begin{array}{c}\mathrm{T}_{3} \mathrm{M}_{1} \mathrm{~F}_{1} \mathrm{P}_{1} \mathrm{D}_{1} \mathrm{~V}_{2} \\
(3.955)\end{array}$ & 0.57 & 48.7 \\
\hline $\begin{array}{l}\text { Logistics } \\
\text { distribution }\end{array}$ & $\&$ & $\begin{array}{c}\mathrm{T}_{3} \mathrm{M}_{1} \mathrm{~F}_{2} \mathrm{P}_{2} \mathrm{D}_{3} \mathrm{~V}_{1} \\
(2.519)\end{array}$ & $\begin{array}{c}\mathrm{T}_{2} \mathrm{M}_{1} \mathrm{~F}_{2} \mathrm{P}_{1} \mathrm{D}_{2} \mathrm{~V}_{1} \\
(4.432)\end{array}$ & 0.568 & 27.0 \\
\hline
\end{tabular}

\section{Discussions and Conclusions}

This research study tried to examine the perception of three broad managerial groups of Halidia Petrochemicals Limited regarding the adaptation of technology. The dynamism of the SMEs was mapped by the perception of the managerial groups namely, Operations, Marketing, Product development \& Innovation for the absorption of technological change.

Achievement Index (Al), as perceived by the senior managers of all sub units of Marketing Division, is nearly 80 percent. It is significant that export division and business development units has given marked emphasis on the technology branding ( more than 40 percent), may be due to the presence of global players and also understanding competitors strong emphasis on technology upgradation. Eastern region market, as still dominated by HPL, they may be reluctant for adapting new technology assessing the present steady business status.

The sub units of Product Development and Innovation units have established a difference in their perception of the achievement of the SMEs in the adaptation in the technology. Market research team and New Product Innovation team has identified the potentiality of the growth with the new process and new 
product and they also provide the guidelines of the development for technology adaptation. But, in reality, Application-Research -Development ( ARD) Team and Entrepreneurs Training and Development Team has faced the resistance from the units of SMEs as they are generally reluctant to accept new technology as they are expensive and more importantly, it leads to change the whole business process resulting in destruction of the traditional way of doing business.

The senior officials of both of the sub units of Product Development and Innovation have perceived that the achievement made by the SME units with respect to the market dynamism is comparatively low (around 50-60 percent). It is also revealed from the study that the issues associated with the capability enhancement such as thrust on the technology development, flexibility in the production system, understanding the new technology and its implementation has received not much attention as the level of importance of the innovation strategies are around only 30 percent.

The senior managers of Operations Division, having three verticals namely, Materials Management, Production \& Operations Management and Logistics \& Distribution units, has perceived that the operational efficiency in terms of their technological capability is around 55-60 percent. Materials Management sub unit has indicated the importance of the operational efficiency is quite low (17 percent) and it is justified as almost all of the SME units has high level of human inventory and low productivity. It is quite evidenced from the present research of Retkoceri \& Kurteshi (2018) that traditional operational practices have appeared to be among the main barriers to innovation and moves towards the modern production practices.

In this paper, the relative importance of the capability parameters namely, Marketing, Product Development \& Innovation and Operations has been estimated. Based on the estimation, the calculated Achievement Index (AI) has indicated the extent of technological superiority achieved by the SME units and also, the gap present in each of broad dimensions has also worked out.

\section{References}

[1] Alvarez, S.A. \& Barney, J.B. (2001). How Entrepreneurial Firms Can Benefit from Alliances with Large Partners, The Academy of Management Executive, 15(1) pp 139-148.

[2] ASSOCHAM report: April,2015: http://assocham.org/newsdetail.php?id=4937 
[3] Centre for Market Research \& Social Development: March,2015: $\quad$ https://www.packworld.com/article/trends-and-issues/global/indianpackaging-industry-turnover-reach-32-billion-2020

[4] Dess, G., G., Lumpkin, \& Covin, J. (1997). Entrepreneurial Strategy Making and Firm Performance: Tests of Contingency and Configuration Models, Strategic Management Journal 18(1) pp. 2-23.

[5] FICCI report: 2013Retrived from: http://ficci.in/sector/88/Project_docs/sector-profile-FICCIpetrochemical.pdf dated 12-02-2018

[6] Haldia Petrochemicals: http://www.haldiapetrochemicals.com/ dated 15-02-2018

[7] International Finance Corporation, World Bank Group. (2012). Micro, Small and Medium Enterprise Finance in India - A Research Study on Need, Gaps and Way Forward. International Finance Corporation (World Bank Group). Retrieved 11-5-2013, from http://www.ifc.org/wps/wcm/connect/4760ee004ec65f44a165bd45b400a808/MSME+ Report-03-01-2013.pdf?MOD=AJPERES

[8] Knight, G. (2000) Entrepreneurship and Marketing Strategy: The SME under Globalization Journal of International Marketing, 8(2) pp. 12-32.

[9] Miller, D., \& Friesen, P. (1984). Organizations: A Quantum View. Englewood Cliffs, NJ: Prentice Hall.

[10] Ministry of MSME, Government of India. (2013). Annual Report - MSME 2012-13. New Delhi: Ministry of MSME, Government of India

[11] Organization for Economic Co-operation and Development (1997), Globalization and Small and Medium Enterprises (SMEs). Paris Organization for Economic Co-operation and Development. Organization for Economic Co-operation and Development (2000) Small and Medium-sized Enterprises: Local Strength, Global Reach Paris Organization for Economic Cooperation and Development

[12] Porter, M. E. (1980). Competitive Strategy: Techniques for Analyzing Industries and Competitors, Free Press, New York.

[13] Retkoceri, B., \& Kurteshi, R. (2018). Barriers to innovation in services and manufacturing firms: The Case of Kosovo, Holistica 9(2) pp. 73-94

[14] Som, A. (2008). Organizational Re-design and Innovative HRM. Oxford University Press. 